\title{
Discovery of chemosynthesis-based association on the Cretaceous basal leatherback sea turtle from Japan
}

\author{
Robert G. Jenkins, Andrzej Kaim, Kei Sato, Kazuhiro Moriya, Yoshinori Hikida, and Ren Hirayama \\ Acta Palaeontologica Polonica 62 (4), 2017: 683-690 doi:https://doi.org/10.4202/app.00405.2017
}

We report a Late Cretaceous chemosynthetic community fueled by decomposing basal leatherback sea turtle on the ocean floor in the western Pacific. The fossil association representing this community has been recovered from the matrix of a concretion containing a single carapace of Mesodermochelys sp. from Late Cretaceous outer shelf to upper slope deposit of northern Hokkaido, Japan. The carapace displays boreholes most likely performed by boring bivalves, and is associated with molluscan shells, mainly Provanna cf. nakagawensis and Thyasira tanabei. Since this association is similar to fauna already known from Late Cretaceous hydrocarbon seeps, sunken wood, and plesiosaur-falls in Hokkaido, it is suggested that all types of chemosynthesis-based communities in the Late Cretaceous of western Pacific may have belonged to the same regional pool of animals and were not yet fully differentiated into three independent types of communities as it is known today. This finding also indicates that the sulfophilic stage of the vertebrate-fall communities was supported not only by plesiosaur carcasses, which were previously reported, but also by sea turtle carcasses. It highlights the possibility of surviving vertebrate-fall communities through the end-Cretaceous mass extinction event on carcasses of sea turtles which are the only large marine vertebrates surviving this event.

Key words: Dermochelyoidae, Provannidae, Thyasiridae, vertebrate-fall, chemosynthesis-based ecosystem, Cretaceous, Japan.

Robert G. Jenkins [robertgj@staff.kanazawa-u.ac.jp], College of Science and Engineering, Kanazawa University, Kanazawa 920-1192, Japan. Andrzej Kaim [kaim@twarda.pan.pl ], Institute of Paleobiology, Polish Academy of Sciences, ul. Twarda 51/55, 00-818 Warszawa, Poland. Kei Sato [keisato@kueps.kyoto-u.ac.jp], Faculty of Science, Kyoto University, Kitashirakawaoiwake-cho, Sakyo-ku, Kyoto 606-8502, Japan. Kazuhiro Moriya [kazuhiro-moriya@asahi-koh.co.jp ], Asahi Kohmatsushiryo LTD. Partnership Shin-Takine Mine, Ohgoe, Tamura-city, Fukushima 963-4111, Japan. Yoshinori Hikida [nmhikida@coral.ocn.ne.jp ], Nakagawa Museum of Natural History, Yasukawa 28-9, Nakagawa Town, Hokkaido 098-2626, Japan. Ren Hirayama [renhirayama@gmail.com], School of International Liberal Studies, Waseda University, Nishiwaseda 1-6-1, Shinjuku-ku, Tokyo 169-8050, Japan. 
This is an open-access article distributed under the terms of the Creative Commons Attribution License (for details please see creativecommons.org), which permits unrestricted use, distribution, and reproduction in any medium, provided the original author and source are credited.

FaF 